\title{
Determining Partial Atomic Charges for Liquid Water: Assessing Electronic Structure and Charge Models
}

\author{
Bowen Han, Christine M. Isborn, ${ }^{*}$ and Liang Shi* \\ Chemistry and Chemical Biology, University of California Merced, Merced, California \\ 95343, USA \\ E-mail: cisborn@ucmerced.edu; Ishi4@ucmerced.edu
}

\begin{abstract}
Partial atomic charges provide an intuitive and efficient way to describe the charge distribution and the resulting intermolecular electrostatic interactions in liquid water. Many charge models exist and it is unclear which model provides the best assignment of partial atomic charges in response to the local molecular environment. In this work, we systematically scrutinize various electronic structure methods and charge models (Mulliken, Natural Population Analysis, CHelpG, RESP, Hirshfeld, Iterative Hirshfeld, and Bader) by evaluating their performance in predicting the dipole moments of isolated water, water clusters, and liquid water as well as charge transfer in the water dimer and liquid water. Although none of the seven charge models is capable of fully capturing the dipole moment increase from isolated water $(1.85 \mathrm{D})$ to liquid water (about $2.9 \mathrm{D}$ ), the Iterative Hirshfeld method performs best for liquid water, reproducing its experimental average molecular dipole moment, yielding a reasonable amount of intermolecular charge transfer, and showing modest sensitivity to the local water environment. The performance of the charge model is dependent on the
\end{abstract}


choice of the density functional and the quantum treatment of the environment. The computed molecular dipole moment of water generally increases with the percentage of the exact Hartree-Fock exchange in the functional, whereas the amount of charge transfer between molecules decreases. For liquid water, including two full solvation shells of surrounding water molecules (within about $5.5 \AA$ of the central water) in the quantum-chemical calculation converges the charges of the central water molecule. Our final pragmatic quantum-chemical charge assigning protocol for liquid water is the Iterative Hirshfeld method with M06-HF/aug-cc-pVDZ and a quantum region cutoff radius of $5.5 \AA$. 


\section{Introduction}

Partial atomic charges are widely used to model electrostatic interactions in molecular simulations. However, the partial atomic charge is not an observable and its assignment is ambiguous. Moreover, the partial atomic charge depends on the local environment of the atom and should vary as the electronic density re-distributes within the molecule and between molecules, sometimes termed polarization and charge transfer, respectively. For example, an ion in aqueous solution may carry a different net charge from its formal charge due to the charge transfer between the ion and water. ${ }^{1-4}$ A plethora of methods have been proposed to assign partial atomic charges and the goal of this work is to devise a quantum-chemical protocol for assigning variable partial atomic charges for condensed phases that is responsive to the local molecular environment, using water as an example.

The strong intermolecular hydrogen bond in water leads to significant charge redistribution, including both polarization and charge transfer. This electronic redistribution is seen by the large increase in dipole moment: the dipole moment of an isolated water molecule is $1.855 \mathrm{D},{ }^{5}$ whereas the dipole moment in liquid water increases greatly to about $2.9 \mathrm{D},{ }^{6,7}$ indicating substantial mutual polarization between water molecules. Charge transfer also takes place upon hydrogen bond formation and is believed to be responsible for water surface charging $^{8-10}$ and some peaks in the low-frequency IR $^{11-19}$ and Raman spectra. ${ }^{20-22}$ Assigning fixed partial atomic charges to water has been a popular practice in modeling water in simulations, and these so-called non-polarizable water models, ${ }^{23-29}$ often parameterized empirically, have shown great success in reproducing a wide range of experimental properties of water, ${ }^{29}$ including its sophisticated phase diagram, while failing in scenarios that call for

flexible partial atomic charges, such as the low-frequency region of the IR spectrum ${ }^{30-33}$ and water in heterogeneous environments. ${ }^{34-37}$

Charge models can be grouped into different categories with very different philosophies. ${ }^{38-41}$ Because we are pursuing a method that can accommodate polarization and charge transfer, we base our protocol on quantum-chemical calculations, and therefore focus on Class 
II and Class III charge models following the classification of Cramer in Ref. 38. Charge models in Class II partition the wave function into atomic orbitals and different choices of atomic orbitals lead to different charge models, such as Mulliken ${ }^{42}$ and natural population analy$\operatorname{sis}^{43,44}$ (NPA). Among other issues, these orbital-based methods are often overly-sensitive to basis set. ${ }^{45-48} \mathrm{NPA}$ is more stable to changes in the basis set than Mulliken, ${ }^{47}$ but NPA also tends to over-estimate the magnitude of the charges. ${ }^{47,48}$ Charge models in Class III determine charges based on analysis of some physical observable from quantum-chemical calculations, such as the electrostatic potential (ESP) or the electron density. ESP-based methods, e.g., $\mathrm{CHelpG}^{49}$ (i.e., charges from electrostatic potentials using a grid), aim to find the optimal partial atomic charges to reproduce the ESP at grid points around the molecule. ESP charges are useful in modeling electrostatic interaction in molecular simulations, ${ }^{50-54}$ but are known to be problematic for flexible molecules and large molecules. ${ }^{49,55-57,57,58}$ The issue with flexible molecules can be addressed with the restrained ESP ${ }^{59}$ (RESP) method, however unphysical charges may be generated for atoms buried inside large molecules even using RESP. ${ }^{40,60}$ Electron-density based methods differ in their ways of spatially partitioning the electron density; for example, Hirshfeld ${ }^{61}$ and many of its variants ${ }^{62-67}$ (e.g., Iterative Hirshfeld $^{62}$ ) make use of the electron densities of constituent atoms to partition the molecular density and Bader charge analysis ${ }^{68}$ divides space according to zero-flux surfaces in the electron density.

The performance of many of the aforementioned charge models have been assessed on isolated water. ${ }^{47,69-72}$ An early study by Åstrand and co-workers found that the Mulliken charges are highly sensitive to the choice of basis set and show no convergence with respect to basis set size for water. ${ }^{69}$ Bickelhaupt and co-workers comprehensively examined the charge predictions from Mulliken, Bader, Hirshfeld, NPA, and Voronoi deformation density $(\mathrm{VDD})^{47}$ charge models on a wide range of small molecules including water, finding that Hirshfeld and VDD models yield similar and physically meaningful charges. ${ }^{47}$ Martin and Zipse focused on the charge prediction for isolated water using six charge models including 
Mulliken, NPA, Bader, CHelpG, Merz-Kollman ${ }^{58,73}$ (MK), and RESP. ${ }^{70}$ They found that ESP-based methods (CHelpG, MK, RESP) accurately reproduce the dipole moment of an isolated water molecule, whereas NPA and Bader significantly overestimate it. Despite these benchmark studies on isolated water, it remains largely unknown which charge models are suitable for assigning partial atomic charges to water in the condensed phase.

In this work we determine a quantum-chemical protocol to assign partial atomic charges to water by benchmarking quantum-chemical methods and charge models against the dipole of an isolated water molecule, of small water clusters, and of many configurations of liquid water, and charge transfer in water dimer and liquid water. For the sake of computational efficiency, we consider density functional theory (DFT), comparing with correlated wavefunction methods, namely CCSD and MP2, for smaller clusters. Available experimental data and the CCSD results are used to guide our choices of density functional, basis set, and charge model. The rest of the paper is organized as follows. In Sec. 2, we present the details of the quantum-chemical calculations and molecular dynamics (MD) simulations. In Sec. 3, we assess the performance of different quantum chemistry methods, basis sets, and charge models for an isolated water molecule, water clusters, and liquid water configurations. The effects of non-local Hartree-Fock exchange on the computed dipole moment and charge transfer are also discussed. In Sec. 4, we conclude with our recommended protocol of assigning partial atomic charges for liquid water.

\section{Computational methods}

Various wave function methods, density functionals, and basis sets are benchmarked against the experimental dipole moment of a single water molecule with a small subset of these methods then used for the water cluster and liquid water calculations. All quantum chemical calculations of electron densities were performed using the Gaussian 16 program ${ }^{74}$ with default integration grids and convergence settings. The wave function methods included 
Hartree-Fock (HF), MP2, and CCSD calculations. The density functionals tested were (1) BLYP, ${ }^{75,76}$ a generalized gradient approximation (GGA) functional; (2) B3LYP, ${ }^{76,77}$ a hybrid GGA functional with 20\% HF exact exchange; (3) M06-2X, ${ }^{78}$ a hybrid meta-GGA with 54\% HF exchange; (4) M06-HF, ${ }^{79,80}$ another hybrid meta-GGA with 100\% HF exchange; (5) $\omega$ B97X-D: ${ }^{81}$ a long-range corrected hybrid functional with dispersion correction and a rangeseparation parameter of $\omega=0.2 ;(6) \mathrm{LC}-\omega \mathrm{PBE},{ }^{82}$ a long-range corrected hybrid functional with $\omega=0.4$; and (7) B2PLYP, ${ }^{83}$ a double hybrid functional with $53 \% \mathrm{HF}$ exchange. The basis sets surveyed are: STO-3G, 3-21G, 6-31G, 6-31G(d),6-31G(d,p), 6-311G(d,p), 6-311++G(3df,3pd), cc-pVDZ, cc-pVTZ, cc-pVQZ, cc-pV5Z, aug-cc-pVDZ, aug-cc-pVTZ, aug-cc-pVQZ, aug-cc-pV5Z. No attempt was made to extrapolate to the complete basis set limit.

Seven charge models are considered in this work: Mulliken, NPA, CHelpG, RESP (using CHelpG grids), Bader, Hirshfeld, and Iterative Hirshfeld. The Iterative Hirshfeld method treats the atomic density as the iteration variable to converge (fractional) atomic population, whose corresponding atomic density may be represented as the ensemble average of those from neutral and ionic forms of the element. ${ }^{62}$ This iterative treatment eliminates the arbitrariness in the choice of the reference promolecule and provides more reasonable (usually larger) charges than the original Hirshfeld method. ${ }^{62}$ Mulliken, NPA, CHelpG, and Hirshfeld charges were obtained using the Gaussian16 program, whereas Bader, RESP, and Iterative Hirshfeld charges were generated with the Multiwfn program ${ }^{84}$ using Gaussian16 output files. The computational cost of Bader charges becomes significant with the larger water clusters. The Q-Chem program ${ }^{85}$ was used to verify the Iterative Hirshfeld results.

The geometry used in all calculations on a single water molecule is the experimental gas phase geometry ${ }^{86}$ with $r_{O H}=0.95781 \AA$ and $a_{H O H}=104.4776^{\circ}$. The calculated dipole moments are compared to the experimental value, $1.855 \mathrm{D} .{ }^{5}$ For the calculations on water clusters, we consider the water dimer and cyclic water clusters up to hexamer. We adopt the geometries optimized by Miliordos and Xantheas ${ }^{87}$ at the level of CCSD(T) with aug- 
cc-pV5Z for dimer, aug-cc-pVQZ for trimer and tetramer, and aug-cc-pVDZ for pentamer and hexamer. The dimer geometry is in excellent agreement with experiment, ${ }^{88}$ and each water molecule in the cyclic water clusters donates one hydrogen bond and accepts one hydrogen bond to allow the highest possible point-group symmetry for the clusters (i.e., $C_{1}$ for trimer and pentamer, $S_{4}$ for tetramer, and $S_{6}$ for hexamer). To obtain liquid water configurations for quantum chemical calculations, a classical MD simulation of liquid water was performed with the TIP4P/2005 27 water model using the GROMACS version 2018.4 package. ${ }^{89,90}$ The rigid TIP4P/2005 model has the experimental gas-phase geometry and reproduces a wide range of experimental properties of liquid water, ${ }^{27,29}$ including liquid densities and radial distribution functions, though it fails to predict the static dielectric constant $^{27}$ and low-frequency infrared spectrum of liquid water. ${ }^{22,91}$ A cubic simulation box with 2000 water molecules was simulated in the NPT ensemble at $298 \mathrm{~K}$ and 1 bar using a Nosé-Hoover thermostat ${ }^{92,93}$ and Parrinello-Rahman barostat ${ }^{94}$ with a coupling constant of 0.5 ps. The velocity Verlet integrator with a time step of 1 fs was used in the MD simulation. A cutoff of $1.0 \mathrm{~nm}$ was applied to the Lennard-Jones interactions and the energy and pressure were corrected. The electrostatic interactions were computed using the Particle-Mesh-Ewald $\operatorname{method}^{95,96}$ with a Coulomb cut-off of $1.0 \mathrm{~nm}$. The 1000 configurations used for liquid water were randomly selected from a 20-ns trajectory with the configurations saved every 1 ps.

The partial atomic charges were used to compute the dipole moment. For any individual water molecule within clusters and liquid water, very likely the molecule is no longer neutral due to charge transfer, so when computing its molecular dipole moment, the origin is chosen to be the center of its nuclear charges. For liquid water, where we wish to account for the possibility of charge transfer between water molecules, a water molecule was randomly chosen from an MD configuration as the central water molecule. Any water molecules within a cutoff radius of the central water based on oxygen-oxygen (O-O) distance are treated explicitly in the quantum mechanical (QM) calculation, with the remaining water molecules in the MD configuration included as molecular mechanical (MM) point charges, specifically those from 
TIP4P/2005 water model. This type of QM/MM calculation was performed to obtain the partial atomic charges and molecular dipole moment of the central water molecule. The cutoff radii of the QM region considered in this work were $3.3 \AA, 5.5 \AA, 8.0 \AA$, and 10.0 $\AA$, which on average correspond to $5,23,73$, and 143 water molecules in the QM region, respectively. The cutoffs of $3.3 \AA$ and $5.5 \AA$ were chosen to roughly match the minima after the first and second peaks, respectively, in the $\mathrm{O}-\mathrm{O}$ radial distribution function of liquid water at ambient conditions using TIP4P/2005.

\section{Results and discussion}

Our goal in this work is to determine a practical protocol for assigning partial atomic charges to liquid water structures, allowing for the possibility of intramolecular polarization and intermolecular charge transfer while reproducing the experimental dipole moment. First we examine the accuracy of a variety of electronic structure methods, basis sets, and charge models in predicting the dipole moment of a single water molecule by comparing to the experimental value. Then we assess the trends of density functionals and charge models on water clusters of increasing size (dimer to hexamer). Finally, we investigate the behavior of liquid water by testing how the molecular dipole varies with QM region size, comparing the dipole to the experimental value, and analyzing the distribution of molecular charges and dipole moments.

\subsection{Isolated water molecule}

For a single water molecule in vacuum, the results of both the electron density-based dipole moment and that computed from partial atomic charges for all the combinations of electronic structure methods, basis sets, and charge models considered in this work are tabulated in the Supporting Information (SI, Tables S1 - S10). Trends in electronic structure methods

and basis set are consistent with those found by Hait and Head-Gordon. ${ }^{97}$ To focus on the 
moderate to large size basis set results, the percent error of the quantum mechanical electron density-based dipole moment compared to the experimental value (1.85 D) is shown in Fig. 1 for cc-pVDZ, aug-cc-pVDZ, and aug-cc-pV5Z basis sets. For almost all electronic structure methods, the dipole moment is overestimated and the addition of diffuse functions decreases the dipole moment, suggesting the importance of diffuse functions in accurately modeling the molecular dipole. Increasing the basis set size from aug-cc-pVDZ to aug-cc-pV5Z does not substantially improve the accuracy, and for M06-HF the error increases, a trend also noted by Hait and Head-Gordon. ${ }^{97}$ HF performs the worst with a percent error of over $7 \%$, due to the well-known overestimation of dipole moments by HF theory. ${ }^{97-99}$ The CCSD method gives less than $1 \%$ error in dipole moment with augmented basis sets; with both MP2 and CCSD results much improved over the HF results, electron correlation certainly improves the description of the electron density of water.

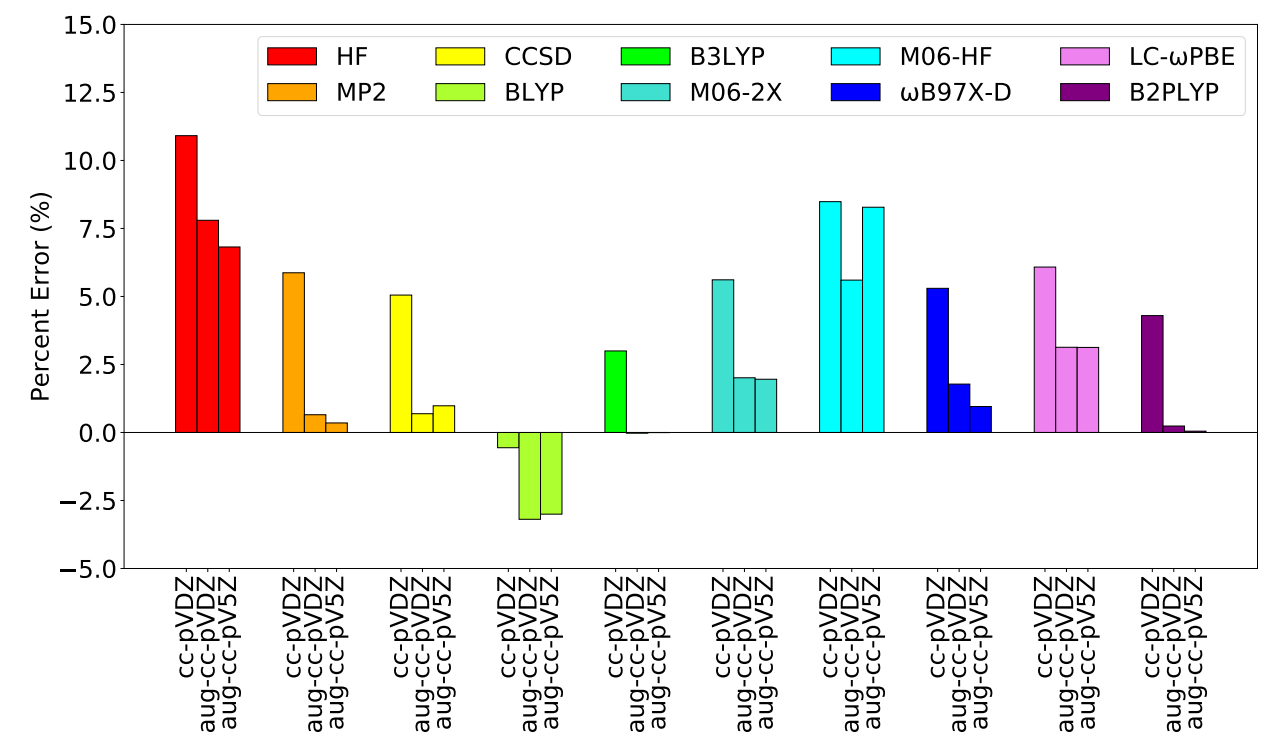

Figure 1: Percent error of the calculated dipole moment of a single water molecule as predicted by the quantum mechanical calculation compared to the experimental value (1.855 D). Experimental geometry of water is used in the calculation.

For the density functionals, we see that the molecular dipole moment increases with the 
increasing percentage of exact exchange. The BLYP functional has zero exact exchange and is the only method that underestimates the dipole moment, whereas M06-2X and M06-HF overestimate the dipole moment, and B3LYP reproduces the experimental dipole moment almost perfectly with augmented basis sets. For the long-range corrected functionals, the percent error of $\omega \mathrm{B} 97 \mathrm{X}-\mathrm{D}$ is less than $2 \%$ with augmented basis sets, and that of LC- $\omega \mathrm{PBE}$ is about 3\% with augmented basis sets. B2PLYP, a double hybrid functional, provides higher accuracy due to the inclusion of MP2 correlation; however, because of the high computational cost, B2PLYP will be difficult to use in the larger electronic structure calculations of liquid water. Accurately computing the electron density and the resulting dipole moment of a water molecule therefore clearly requires a proper balance of correlation and exact exchange.

The percent error of the dipole moment of a single water molecule calculated from partial atomic charges compared to the experimental value is shown in Fig. 2 for different charge models and selected quantum chemical methods (MP2, CCSD, BLYP, B3LYP, M06-2X, and M06-HF) with the aug-cc-pVDZ basis set. There is substantially more variation in computed dipole moment across charge models than across electronic structure methods. We take CCSD as the standard to assess the performance of the seven charge models because the electron density from CCSD/aug-cc-pVDZ accurately reproduces the experimental dipole of water with a relative error of less than $1 \%$ (see Fig. 1). As might be expected, the dipole prediction from the point charges is (much) worse than that computed directly from the CCSD electron density, regardless of the charge model. Among the seven charge models, the ESP based methods, CHelpG and RESP, clearly outperform other methods (only 3.5\% relative error) due to deriving partial atomic charges directly from the quantum chemical electrostatic potential. The additional restraints imposed on charges in the RESP method do not affect the results. The Mulliken charges, known to be highly sensitive to the basis set, significantly underestimate the dipole moment of water by $67 \%$, whereas NPA overestimates it by $43 \%$. This finding is consistent with the QCISD results in the study by Martin and Zipse. ${ }^{70}$ Because these orbital-based charge models assign charges to orbitals associated 
with atoms, they do not necessarily reflect the spatial distribution of the electron density that governs the molecular dipole. Among the three methods that directly use the electron density, the Bader method almost doubles the experimental value, predicting too much ionic character for the covalent $\mathrm{OH}$ bond in water. This is a well-known flaw ${ }^{47}$ of Bader charges due to the use of electron-density zero-flux dividing surfaces, which leads to unphysically large space partitioning for oxygen in water. The Hirshfeld method partitions the electron density based on spherically averaged atomic densities, naturally accounting for the sizes of atoms in the molecule. However, as is often the case with Hirshfeld, ${ }^{100}$ it substantially underestimates the dipole, here by over $50 \%$. Iterative Hirshfeld, developed to alleviate this issue by optimizing the atomic densities via an iterative procedure, increases the magnitude of the partial atomic charges and molecular dipole, but in the case of the water monomer it over-corrects, predicting a dipole magnitude about 30\% higher than the experimental one.

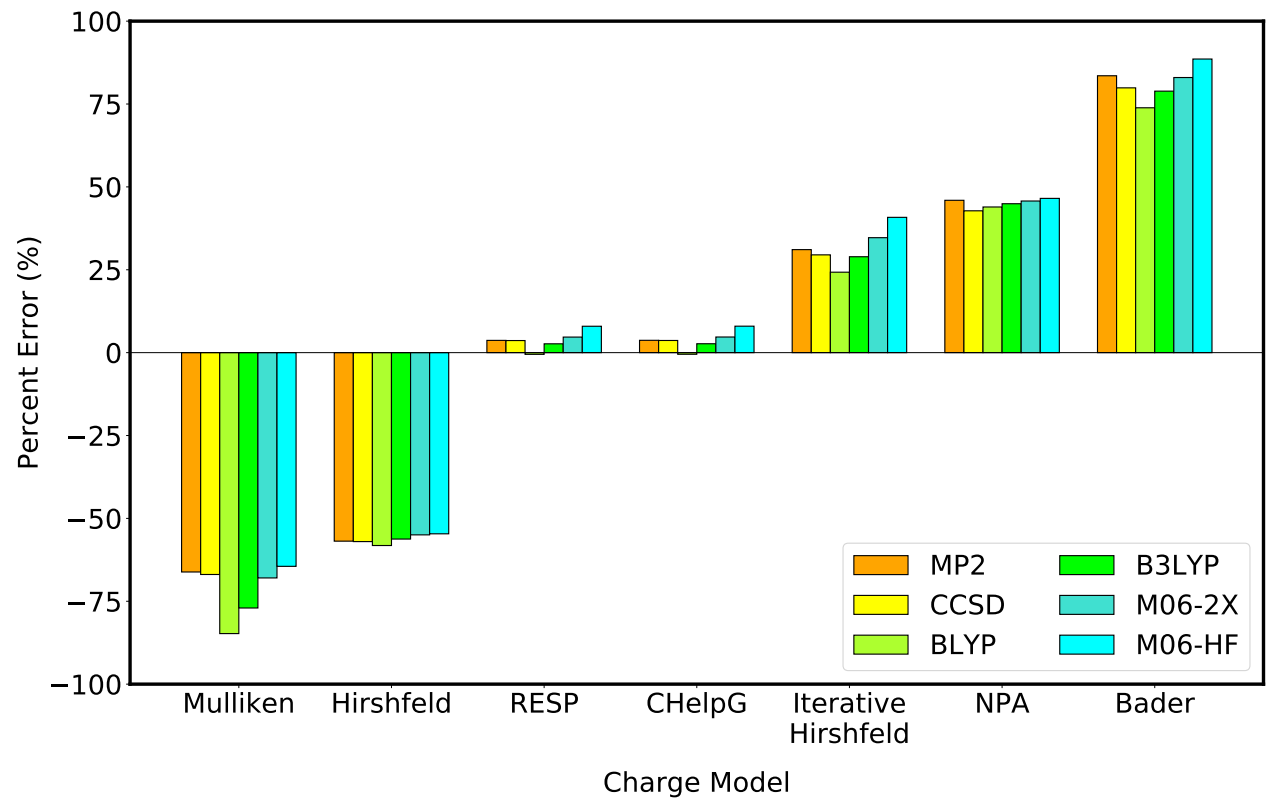

Figure 2: Percent error of the dipole moment of a single water molecule calculated from partial atomic charges compared to the experimental value $(1.855 \mathrm{D})$. The aug-cc-pVDZ basis set is used for all electronic structure calculations on the experimental geometry of water. 
The predicted oxygen charges and molecular dipoles of the single water molecule from the charge models with all the electronic structure methods and basis sets considered in this work are summarized in Tables S1-S10 of the SI. Overall, for the isolated water molecule, we find that the error introduced by the charge model dominates the error in electron density from the quantum chemical method. The trends across electronic structure methods from computing the dipole moment from the electron density still hold when computing the dipole moment from partial atomic charges. However, despite the fact that NPA significantly overestimates the water dipole as shown in Fig. 2, this method is fairly insensitive to the amount of $\mathrm{HF}$ exchange in the functional as its predicted dipole increases only by $1.9 \%$ going from BLYP to M06-HF compared to an increase of $8.9 \%$ based on the electron density (see Fig. 1). In contrast, Iterative Hirshfeld is overly sensitive to the amount of the HF exchange, showing an increase of $13.0 \%$ in the water dipole, whereas CHelpG and RESP quantitatively reproduce the increase based on the electron density. Overall, CHelpG and RESP with BLYP/aug-cc-pVDZ give the best agreement with experiment (computed 1.85 D, experiment $1.855 \mathrm{D}$ ); this good agreement is due to error cancellation from BLYP underestimating the dipole moment by $3.2 \%$ from its electron density and CHelpG and RESP charge models slightly overestimating the molecular dipole moment.

\subsection{Water clusters}

The dipole moment of the water molecule increases from 1.855 D for the isolated monomer to $\sim 2.9 \mathrm{D}$ in liquid water due to the polarization by the environment. In order to systematically study such an effect, we consider the water dimer and cyclic water clusters up to hexamer, where the electronic polarization should increase with cluster size. We study the computed individual molecular dipole moments within the cluster, as well as the total dipole moment and charge transfer for the water dimer.

The total dipole moment of the water dimer computed from various electronic structure methods and charge models using aug-cc-pVDZ is summarized in Tables S11-S21 and Fig. 
S2-S3 in the SI, with the trends being very similar to those for isolated water. The experimental dipole moment of the water dimer of $2.643 \mathrm{D}^{88}$ is almost perfectly reproduced by the electron-density based dipole moment from CCSD (2.641 D) and the MP2 dipole moment is also very accurate $(2.649 \mathrm{D})$. The percent errors from density functional methods are all less than 5\% (see Table S11 and Fig. S2 in the SI), with the calculated dipole increasing with the amount of HF exact exchange as seen for the isolated water molecule. The double hybrid functional, B2PLYP, produces an accurate dipole $(2.667 \mathrm{D})$, presumably due to a proper balance between exchange and correlation. As is the case with the isolated water, much larger errors are seen with charge models compared to errors from electronic structure methods (see Fig. S3 in the SI). CHelpG and RESP stand out with the smallest relative errors (2.9\%-7.1\%). The Mulliken and Bader methods significantly under- and over-estimate the dimer dipole by over 50\%, respectively, and the Hirshfeld, Iterative Hirshfeld, and NPA charge models have errors of around $25 \%$. These observations of the water dimer again suggest that the electron density predicted by the electronic structure methods considered here, in particular CCSD, is fairly accurate, but the charge model introduces substantial errors in the prediction of the water dipole.

In the water dimer, the hydrogen bond acceptor (i.e., electron donor) transfers some charge to the hydrogen bond donor (i.e., electron acceptor), rendering the hydrogen bond acceptor (donor) positively (negatively) charged. This charge transfer, along with polarization, is responsible for the weakening of the $\mathrm{OH}$ bond involved in the hydrogen bond, observed as a spectral red-shift in the OH-stretch vibrational spectroscopy of water. ${ }^{101-103}$ Like the partial atomic charges, the amount of charge transfer is not defined unequivocally and its value varies greatly in the literature (e.g., 0.002-0.080e $)^{44,104-107}$ depending on the electronic structure method and charge model. Ronca and co-workers ${ }^{104}$ advocated the use of charge-displacement analysis to quantify charge transfer, where the electron density difference between the interacting and non-interacting water dimers of the same geometry is examined as a function of the separation between the two water molecules. Their electron 


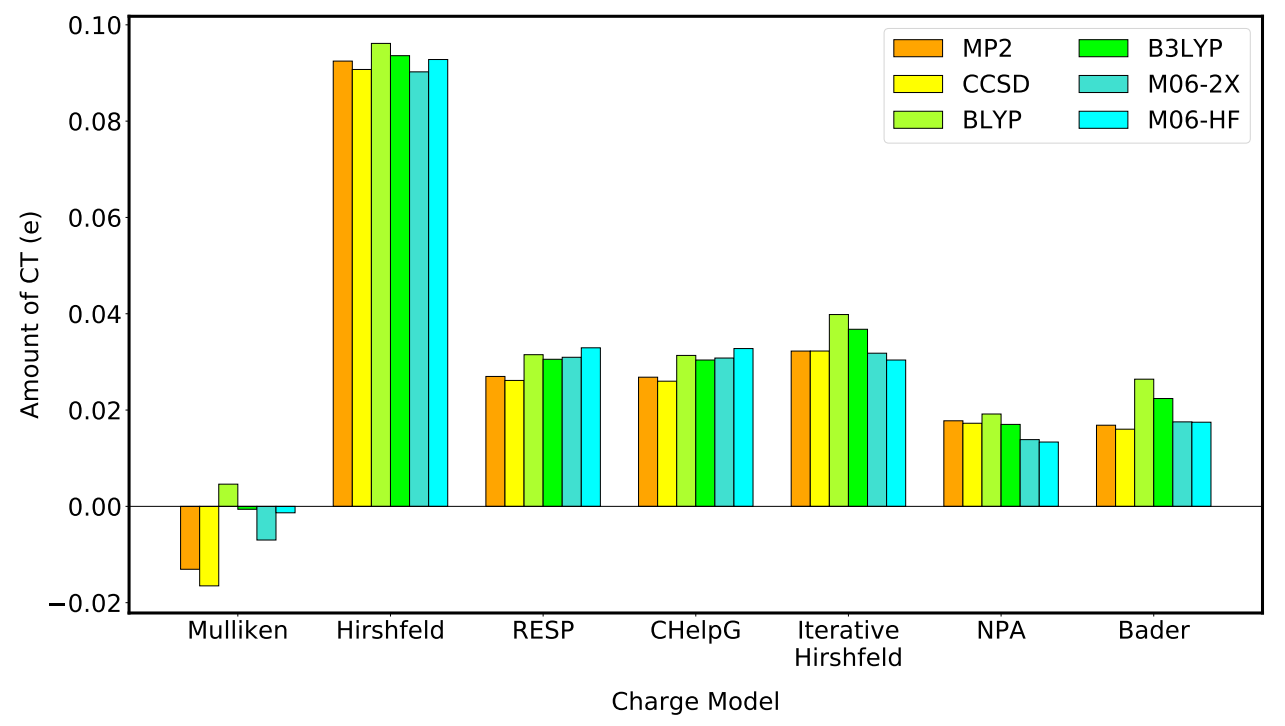

Figure 3: The amount of CT from the hydrogen bond acceptor to donor in the water dimer. The aug-cc-pVDZ basis set is used for all electronic structure calculations.

density based analysis, which does not need a charge model, suggests that the amount of charge transfer in the water dimer is $\sim 0.014 e$ with an upper limit of about 0.018-0.020e. Consistent with their estimates, most of the charge models considered in this work predict the amount of charge transfer for a water dimer in the range of 0.010-0.035e, as shown in Fig. 3. The outliers are the Mulliken and Hirshfeld methods. Mulliken predicts that the hydrogen bond donor (acceptor) is unphysically positively (negatively) charged with CCSD, MP2, HF, and most density functionals considered here. Hirshfeld gives a charge transfer amount of around 0.090e, much larger than those from other methods and previous studies.

For the three electron-density based methods (i.e., Hirshfeld, Iterative Hirshfeld, and Bader), the calculated total dipole moment of the dimer, individual water molecular dipole moments, and the magnitudes of the partial atomic charges increase with the amount of HF exchange in the functional (see Figs. S2-S3 in the SI), whereas the amount of charge transfer decreases (see Fig. 3). This trend is due to the well-known delocalization error: local and semi-local functionals tend to over-emphasize electron delocalization, whereas HF and 
global hybrid functionals with large fractions of HF exchange tend towards over-localization of the electron density. ${ }^{108}$ Stronger electron localization leads to a larger dipole moment and a smaller amount of charge transfer. For the three density-based charge models combined with the density functionals examined here, M06-2X and M06-HF predict an amount of charge transfer between water molecules very close to that from CCSD. Because the total dipole moment of the dimer calculated directly from the electron density increases with the amount of HF exchange (see Fig. S2 in the SI), the charge model should capture this trend. However, Fig. S3 of the SI shows that Hirshfeld and NPA charge models fail to show such a dependence on HF exchange due to their insensitivity to electron density variation, which further manifests in the larger water clusters and liquid water discussed later.

We now proceed to the cyclic water clusters from trimer to hexamer, in which each water molecule donates one hydrogen bond and accepts one hydrogen bond so that all water molecules are polarized in a cooperative manner. The electronic polarization increases with the cluster size, as evidenced by the gradually red-shifted $\mathrm{OH}$-stretch vibrational frequencies observed in both experiment ${ }^{109}$ and quantum chemical calculation. ${ }^{110}$ The water hexamer often serves as the smallest water cluster to understand hydrogen bonding in liquid water and ice, where the cyclic isomer is one of the prominent structural motifs. ${ }^{111-114}$ The cyclic water hexamer $\mathrm{OH}$-stretch frequency (around $3330 \mathrm{~cm}^{-1}$ ) ${ }^{109}$ falls well within the $\mathrm{OH}$-stretch frequency range of liquid water (a broad peak centered around $3400 \mathrm{~cm}^{-1}$ ), ${ }^{12,115}$ indicative of comparable polarization around the $\mathrm{OH}$ bond. Therefore, we expect that with increasing polarization, the molecular dipole moment of water should increase with cluster size, approaching that in liquid water with the cyclic hexamer giving the closest value.

All the calculated results for cyclic water clusters with different electronic structure methods and charge models are given in Tables S22-S61 of the SI. Due to the symmetries of the cyclic water clusters, all the water molecules in each cluster should have almost identical oxygen/hydrogen partial atomic charges, as confirmed in Figs. S4-S7 of the SI, although appreciable variations are seen for the trimer and pentamer with CHelpG and RESP. The 


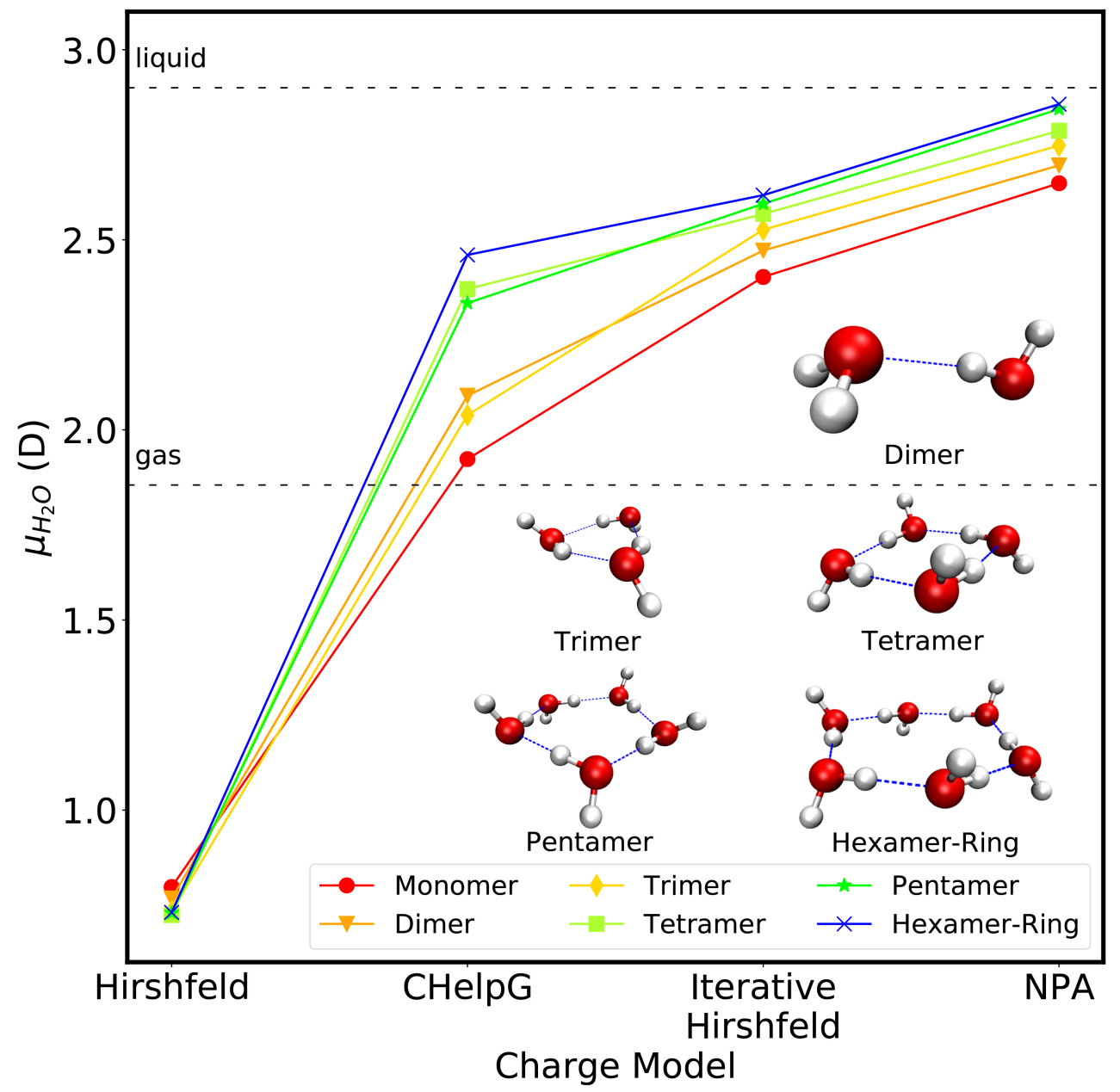

Figure 4: Average water molecular dipole moment in water clusters calculated from partial atomic charges with different charge models using CCSD/aug-cc-pVDZ. The two dashed horizontal lines indicate the experimental dipole moment of the isolated water molecule, and the average water molecular dipole in liquid water estimated from experiment, respectively. 
average molecular dipoles from CCSD/aug-cc-pVDZ and four selected charge models are displayed in Fig. 4 (Mulliken and Bader are not shown for the sake of pictorial clarity, along with the RESP results that are almost identical to CHelpG). The molecular dipole computed for the isolated water (i.e., monomer) to cyclic hexamer gradually increases when using the Iterative Hirshfeld and NPA charge models, suggesting that both methods capture the increasing polarization effects of the cooperative cyclic hydrogen bond network. The Hirshfeld molecular dipoles are unphysically small (even smaller than that of an isolated water molecule) and show very little size dependence, suggesting that Hirshfeld does not correctly capture polarization effects. CHelpG (as well as RESP) predicts that the average molecular dipole in the dimer is greater than that in the trimer. As is the case with isolated water and water dimer, Mulliken significantly underestimates the molecular dipole (less than $1.5 \mathrm{D}$ ), whereas Bader gives the largest dipole among all the charge models (around 3.5 D, see Figs. S4-S7 of the SI). These observations are consistent for other electronic structure methods, as shown in Figs. S4-S8 of the SI.

In Fig. 4, the experimental dipole moment of the isolated water molecule $(1.855 \mathrm{D})$ and the experimental average molecular dipole in liquid water (about $2.9 \mathrm{D}$ ) are shown as two dashed lines. If we assume that the molecular dipole in the hexamer is close to that in liquid water, Fig. 4 suggests that no charge models considered here can faithfully reproduce the experimental dipole increase from gas to liquid phases. Considering the accuracy of CCSD in describing the electron density in the water monomer and dimer, this inaccuracy is not likely to be a failure of the electronic structure methods but more likely is the failure of the charge models to fully capture the increased electronic polarization going from isolated water to water clusters to liquid water. As seen with the monomer and dimer, the magnitude of the molecular dipole from water monomer to hexamer increases with the amount of HF exact exchange in the density functional (see Fig. S8 in the SI), suggesting that the overpolarization provided by increasing the amount of exact exchange might be a practical way to balance the under-polarization of the charge models to improve the description of the water 
dipole moment in liquid water. In fact, this strategy has been adopted in parametrizing partial atomic charges in some force fields, e.g., AMBER charges for amino acids were derived from HF calculations to use HF's over-polarization to roughly account for solvent polarization. ${ }^{116,117}$

Based on our results on the isolated water molecule and water clusters, we only consider CHelpG, Iterative Hirshfeld, and NPA charge models for the calculations on liquid water. Mulliken and Hirshfeld are excluded due to their significant underestimation of dipole moment and unphysical results for charge transfer in water clusters. Bader is abandoned due to its significant overestimation of dipole moment and high computational cost. The results from RESP are always very similar to those from CHelpG, hence no longer considered.

\subsection{Liquid water}

We next examine the computed dipole moment of the water molecule in the liquid phase, with the goal of reproducing the average dipole moment of $\sim 2.9 \mathrm{D}$ while accommodating intermolecular charge transfer between water molecules. We therefore want the method to be computationally affordable for larger water clusters and to have good convergence behavior with increasing QM region size. For the liquid water configurations studied here, we no longer use CCSD, MP2, or B2PLYP, but instead focus on the more computationally feasible DFT methods.

The convergence of the CHelpG, Iterative Hirshfeld, and NPA molecular dipoles with QM region size are shown in Fig. 5 for the same ten randomly chosen MD configurations using the M06-HF/aug-cc-pVDZ method for the QM region. The molecular dipole moments generated from the CHelpG method do not converge as the radius of the QM region increases from $3.3 \AA$ to $10.0 \AA$ (about 5 to 143 water molecules on average) as shown in Fig. 5(a). The poor convergence for CHelpG stems from the fact that ESP based methods only reproduce the potentials outside of the system, leading to inaccurate charges for atoms buried in the center of the QM region. Compared to the dipole moments from CHelpG, the Iterative 

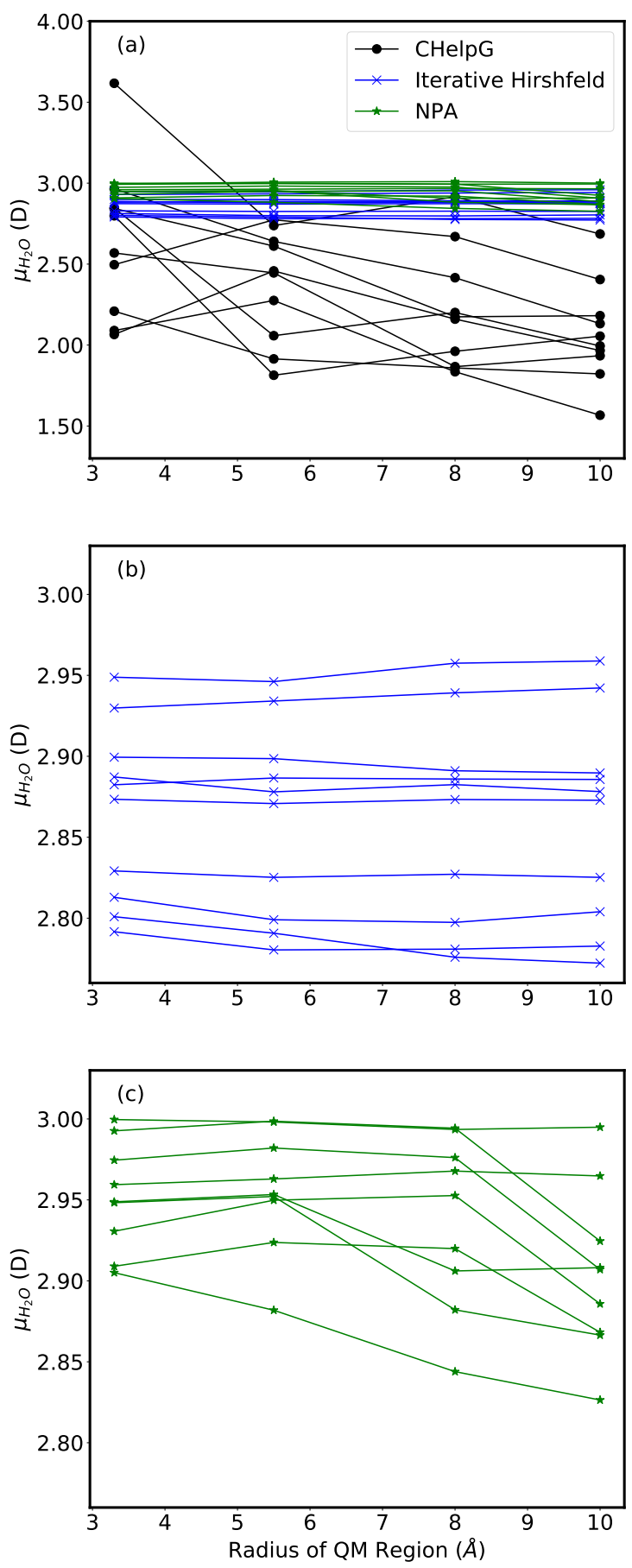

Figure 5: (a) Molecular dipole moment calculated for the same ten MD configurations using the partial atomic charges from CHelpG, Iterative Hirshfeld, and NPA charge models with increasing QM region size. M06-HF/aug-cc-pVDZ is used for the QM region, with TIP4P/2005 MM charges surrounding the QM region. (b) Zoom-in of the Iterative Hirshfeld results. (c) Zoom-in of the NPA results. 
Hirshfeld and NPA results show better convergence with the QM region size with Iterative Hirshfeld being the most stable. Iterative Hirshfeld gives consistent molecular dipoles with increasing QM region size, with a maximum deviation of only $0.02 \mathrm{D}$ going from a QM region radius of $5.5 \AA$ to $10.0 \AA$ (Figure $5(\mathrm{~b})$ ), whereas the NPA predicted dipole varies by as much as $0.07 \mathrm{D}$ for some configurations. Similar trends are observed for other functionals (BLYP, B3LYP, and M06-2X) as shown in Fig. S13 of the SI.

With the QM region radius of $5.5 \AA$, which roughly correspond to two full solvation shells of the central water molecule, we computed the average oxygen charge and average molecular dipole moment of water molecules in liquid water over 1000 randomly chosen MD configurations using CHelpG, Iterative Hirshfeld, and NPA charge models with several functionals and the aug-cc-pVDZ basis set. These results are shown in Fig. 6 with the error bars indicating the ranges of the sampled properties. The oxygen charge from CHelpG is not only much smaller in magnitude compared to those from Iterative Hirshfeld and NPA, but also shows abnormally large variations (e.g., with M06-HF, it ranges approximately from -0.2 to -1.2$)$, reminiscent of the incapability of ESP-based models in predicting charges for large systems. The oxygen charges from both Iterative Hirshfeld and NPA fall within the range of -0.8 to -1.2 , consistent with those used in most empirical water models, ${ }^{23-25,27,28,118}$ and increase in magnitude with the amount of HF exchange in the functional. Consequently, the average molecular dipole in liquid water from Iterative Hirshfeld and NPA also increases with the percentage of HF exchange. For Iterative Hirshfeld, the M06-HF functional most closely reproduces the experimentally estimated molecular dipole (2.9 D) shown as the dashed line in Fig. 6. As is the case with isolated water and water clusters, the oxygen charges and molecular dipoles from NPA show very weak dependence on HF exchange as well as small variations with respect to liquid water configurations, although the predicted average molecular dipole is always fairly close to the experimental estimate. The low sensitivity of NPA to local molecular environment around water is not desirable as liquid water is known to be locally heterogeneous as evidenced by the very broad OH-stretch band in 

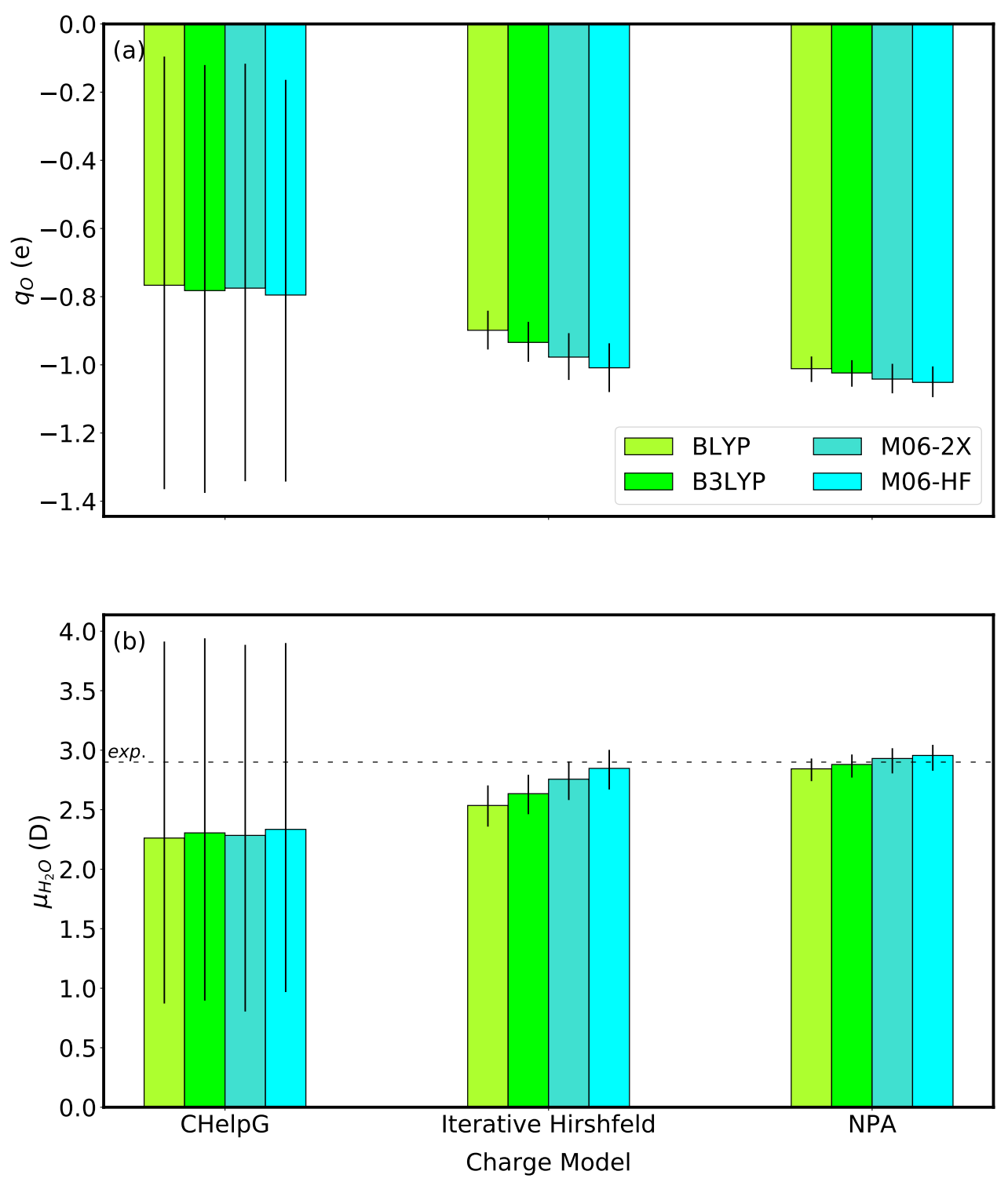

Figure 6: (a) Average oxygen charge and (b) average molecular dipole of water calculated from CHelpG, Iterative Hirshfeld, and NPA charge models using four density functionals (i.e., BLYP, B3LYP, M06-2X and M06-HF) with the aug-cc-pVDZ basis set. The average is obtained over 1000 randomly chosen MD configurations with $5.5 \AA$ as the QM region radius, and the error bar indicates the range of the sampled property. The dashed horizontal line indicates the average water molecular dipole in liquid water estimated from experiment. 
the IR spectrum of isotope-diluted liquid water. ${ }^{115,119}$ In summary, the Iterative Hirshfeld charge model combined with the M06-HF/aug-cc-pVDZ electron density seems to offer the best performance in assigning partial atomic charges for liquid water with a relatively low computational cost, with NPA as a close second with the advantage of giving reasonable charges with non-hybrid functionals.

To further assess the Iterative Hirshfeld model, the distributions of molecular charge and molecular dipole moment using M06-HF/aug-cc-pVDZ with Iterative Hirshfeld are shown in Fig. 7. The same distributions with other functionals are shown in Figs. S14-S15 of the SI. As mentioned earlier, several studies found that the amount of charge transfer between the water molecules in the water dimer is about $0.02 e .^{44,104,106}$ There are efforts to include charge transfer in empirical water models, ${ }^{19,120}$ and the amount of the charge transfer in these studies ranges within $\pm 0.05 e$, consistent with the results from some ab initio MD studies. ${ }^{8,121}$ Our computed net charge of the water molecule in liquid water also falls within this range, as shown in Fig. 7(a). For BLYP and B3LYP functionals, the distributions of molecular charge are slightly broader than those for M06-2X and M06-HF (Fig. S14 of the SI). The reported average molecular dipole moment in liquid water vary widely in the literature: 2.4-3.1 D, depending on theoretical model. ${ }^{32,121-131}$ Our computed distribution of molecular dipole moment in liquid water using M06-HF/aug-cc-pVDZ and Iterative Hirshfeld is shown in Fig. 7 (b), spanning a range from $\sim 2.7 \mathrm{D}$ to $\sim 3.0 \mathrm{D}$. The computed average molecular dipole is $\sim 2.85 \mathrm{D}$, slightly lower than the experimental value of $2.9 \mathrm{D}$, whereas the range of the molecular dipole moment is narrower than some other theoretical studies. ${ }^{32,121-128,130,131}$

Although the protocol of Iterative Hirshfeld charges with the M06-HF/aug-cc-pVDZ electron density can give reasonable partial atomic charges, charge transfer, and molecular dipole for liquid water, we emphasize that none of the charge models considered here is capable of reproducing molecular dipole moments for water going from its gas phase to liquid water. In Fig. S16 of the SI, the computed average molecular dipole moment of water in liquid water is shown along with that for isolated water molecule using the same 

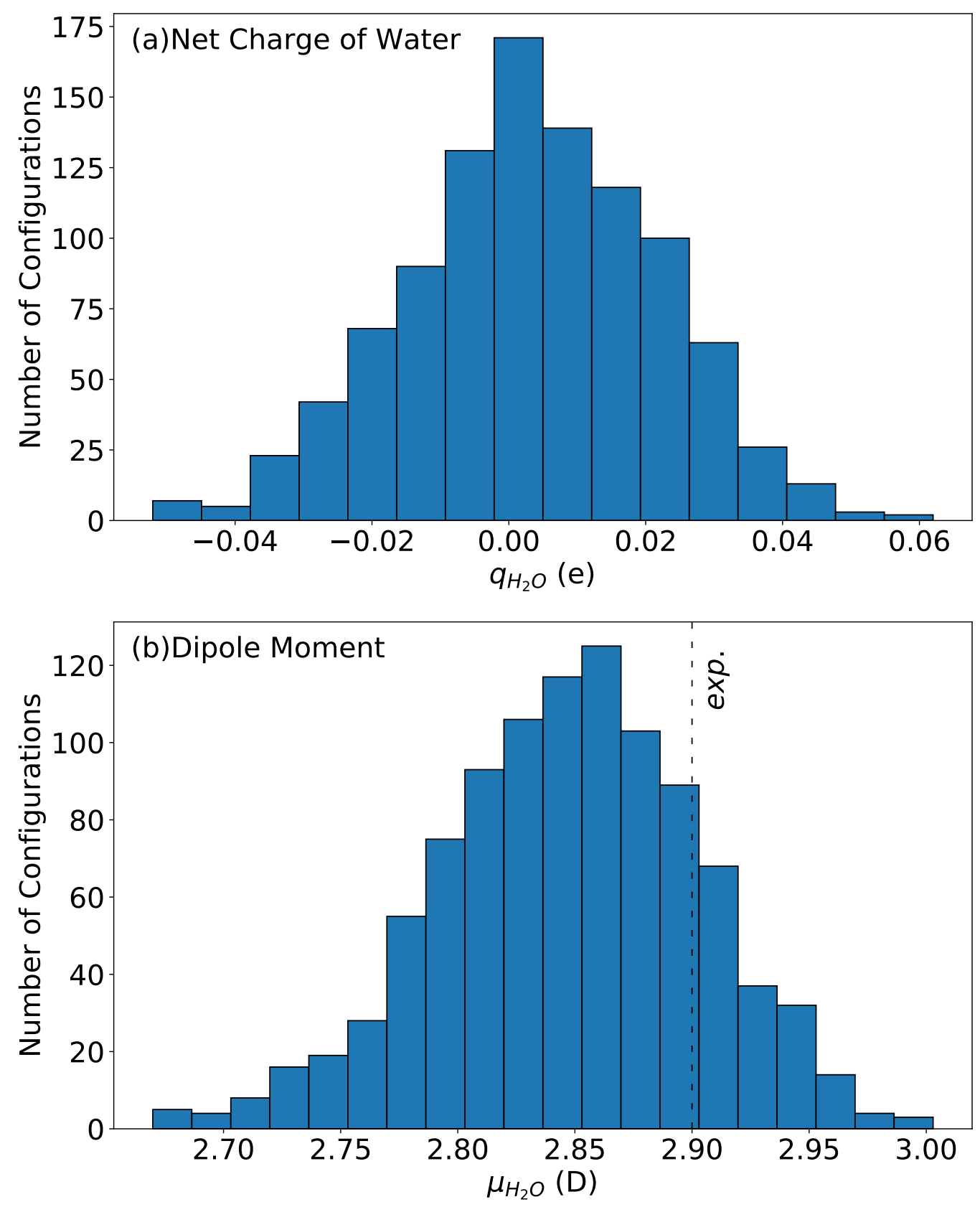

Figure 7: Distribution of net charge and the dipole moment of the central water in liquid phase from the partial atomic charges obtained from Iterative Hirshfeld with M06-HF/augcc-pVDZ and a QM region radius of 5.5 $\AA$. 1000 randomly chosen configurations were used to make the histograms. Dashed vertical line in (b) indicates the experimental molecular dipole moment of water in liquid phase. 
method for the combinations of four functionals and three charge models (Iterative Hirshfeld, CHelpG, and NPA). Experimental values for the molecular dipole moment of water in its gas $(1.855 \mathrm{D})$ and liquid $(2.9 \mathrm{D})$ phases are shown as the dashed horizontal lines. The significant increase of water dipole moment by about $1.0 \mathrm{D}$ is not reproduced by any method, implying that the polarization in liquid water is only partially captured in our calculations. The insufficient polarization from isolated water to liquid is mostly attributed to the failure of the charge models rather than the functionals as ab initio MD simulation with some of these functionals (e.g., BLYP), sometimes including dispersion correction, can reasonably reproduce many properties of liquid water including its IR spectrum. ${ }^{132-135}$ 


\section{Conclusion}

Characterizing liquid water with partial atomic charges is a common practice due to its conceptual simplicity and computational efficiency. However, given the ambiguity in defining partial atomic charge, no consensus exists on a reliable quantum-chemical charge assigning protocol, in particular for liquid water. In this work, we have systematically assessed various electronic-structure methods and charge models by examining their performance in describing the dipole moments of isolated water, water clusters, and liquid water as well as charge transfer in the water dimer and liquid water.

For the isolated water molecule, most of the electronic structure methods examined here can reasonably reproduce the experimental dipole moment based on the electron density and those methods that have a proper balance of correlation and exchange, such as CCSD, MP2, and B2PLYP, give the best predictions. Charge models introduce substantial errors in the predicted dipole with the ESP-based models (i.e., CHelpG and RESP) being the best performers, consistent with the results of Martin and Zipse. ${ }^{70}$ The same trends are observed for the water dimer, where charge transfer is reasonably captured by most charge models. The charge models with a poor description of charge transfer are Mulliken and Hirshfeld, which also predict unphysically small water molecular dipole moments for cyclic water clusters. The anticipated gradual increase of the molecular dipole in the cyclic water clusters with the cluster size is correctly shown by Iterative Hirshfeld and NPA, but not by the ESP-based methods.

In assigning partial atomic charges to liquid water, we find that two full solvation shells of surrounding water molecules are needed to converge the partial atomic charges of the central water molecule assigned by Iterative Hirshfeld or NPA. The CHelpG charge model is not suitable for assigning charges in liquid water as it shows poor convergence with the QM region size and an unphysically large variation in molecular dipole. Despite reproducing the average molecular dipole in liquid water, NPA charges are relatively insensitive to the heterogeneous local environment of water. Our final charge assignment protocol for liquid 
water is Iterative Hirshfeld with M06-HF/aug-cc-pVDZ and a QM region cutoff radius of $5.5 \AA$; this method predicts a molecular dipole distribution for liquid water over a range of about 2.7-3.0 D with an average of 2.85 D, and a net molecular charge distribution within $\pm 0.05 e$, consistent with available experimental estimates and previous calculations.

There are several trends observed over all the water systems considered in this work. The partial atomic charges and dipole moments tend to increase with the percent of exact HF exchange in the functional, whereas the amount of charge transfer decreases. Both Mulliken and Hirshfeld models significantly underestimate the water dipole using the aug-ccpVDZ basis set with Mulliken (Hirshfeld) being overly sensitive (insensitive) to the employed electronic structure method and basis set. The NPA and Iterative Hirshfeld models, which are improved models over Mulliken and Hirshfeld charge models, respectively, tend to give large charges and overestimate dipole moments. Bader always predicts the largest charges and dipole moments among the charge models considered here. CHelpG and RESP can reproduce the molecular dipoles of the isolate water and water dimer, but generate unphysical charges for liquid water. Clearly, none of the seven charge models considered in this work is versatile enough to properly assign water charges for all the water systems; however, with a good understanding of these trends, one can choose a proper combination of quantum chemical method and charge model to find an affordable protocol to assign charges for a specific system. This is the pragmatic approach adopted in this work, which has also been used in some force field parameterizations.

Our final charge assigning protocol for liquid water, Iterative Hirshfeld with M06-HF/augcc-pVDZ and a QM region cutoff radius of $5.5 \AA$, allows charge variation in response to the local environment of water. The application of this protocol can potentially improve the description of the dipole moment surface of liquid water, thereby improving some predicted dielectric properties, such as the low-frequency IR spectrum of liquid water. This protocol is based on the liquid water configurations from TIP4P/2005, arguably the best rigid nonpolarizable water model today. We expect that our protocol can be directly transferred to 
any non-polarizable water model, with slight changes expected in the average dipole and degree of charge transfer due to differences in water configurations. More importantly, the strategy employed in this work for assigning charges will be applicable to other condensedphase systems.

\section{Acknowledgement}

This work was supported by the Department of Energy Basic Energy Sciences CTC and CPIMS programs (Grant No DE-SC0019053). L.S. also acknowledges the support from the University of California Merced start-up funding. This research used computing resources of the National Energy Research Scientific Computing Center (NERSC), a U.S. Department of Energy Office of Science User Facility operated under Contract No. DE-AC02-05CH11231, and the Multi-Environment Computer for Exploration and Discovery (MERCED) cluster at UC Merced under Science Foundation Grant No. ACI-1429783.

\section{Supporting Information Available}

Structures of water clusters; calculated partial atomic charges, molecular dipoles, and net charges if there is charge transfer, for isolated water, water clusters and liquid water using various quantum chemical methods and charge models; and additional figures that compare these results.

\section{References}

(1) Thompson, W. H.; Hynes, J. T. Frequency Shifts in the Hydrogen-Bonded OH Stretch in Halide-Water Clusters. The Importance of Charge Transfer. Journal of the American Chemical Society 2000, 122, 6278-6286.

(2) Cappa, C. D.; Smith, J. D.; Wilson, K. R.; Messer, B. M.; Gilles, M. K.; Cohen, R. C.; 
Saykally, R. J. Effects of Alkali Metal Halide Salts on the Hydrogen Bond Network of Liquid Water. The Journal of Physical Chemistry B 2005, 109, 7046-7052.

(3) Zhao, Z.; Rogers, D. M.; Beck, T. L. Polarization and charge transfer in the hydration of chloride ions. The Journal of Chemical Physics 2010, 132, 014502.

(4) Soniat, M.; Rick, S. W. The effects of charge transfer on the aqueous solvation of ions. The Journal of Chemical Physics 2012, 137, 044511.

(5) Dyke, T. R.; Muenter, J. S. Electric dipole moments of low J states of $\mathrm{H}_{2} \mathrm{O}$ and $\mathrm{D}_{2} \mathrm{O}$. The Journal of Chemical Physics 1973, 59, 3125-3127.

(6) Badyal, Y. S.; Saboungi, M.-L.; Price, D. L.; Shastri, S. D.; Haeffner, D. R.; Soper, A. K. Electron distribution in water. The Journal of Chemical Physics 2000, 112, 9206-9208.

(7) Gubskaya, A. V.; Kusalik, P. G. The total molecular dipole moment for liquid water. The Journal of Chemical Physics 2002, 117, 5290-5302.

(8) Vácha, R.; Marsalek, O.; Willard, A. P.; Bonthuis, D. J.; Netz, R. R.; Jungwirth, P. Charge Transfer between Water Molecules As the Possible Origin of the Observed Charging at the Surface of Pure Water. The Journal of Physical Chemistry Letters 2012, 3, 107-111.

(9) Lee, A. J.; Rick, S. W. Characterizing Charge Transfer at Water Ice Interfaces. The Journal of Physical Chemistry Letters 2012, 3, 3199-3203.

(10) Wick, C. D.; Lee, A. J.; Rick, S. W. How intermolecular charge transfer influences the air-water interface. The Journal of Chemical Physics 2012, 137, 154701.

(11) Zelsmann, H. R. Temperature dependence of the optical constants for liquid $\mathrm{H}_{2} \mathrm{O}$ and $\mathrm{D}_{2} \mathrm{O}$ in the far IR region. Journal of Molecular Structure 1995, 350, 95-114. 
(12) Bertie, J. E.; Lan, Z. Infrared Intensities of Liquids XX: The Intensity of the OH Stretching Band of Liquid Water Revisited, and the Best Current Values of the Optical Constants of $\mathrm{H}_{2} \mathrm{O}(\mathrm{l})$ at $25^{\circ} \mathrm{C}$ between 15,000 and $1 \mathrm{~cm}^{-1}$. Applied Spectroscopy $\mathbf{1 9 9 6}$, $50,1047-1057$.

(13) Afsar, M. N.; Hasted, J. B. Measurements of the optical constants of liquid $\mathrm{H}_{2} \mathrm{O}$ and $\mathrm{D}_{2} \mathrm{O}$ between 6 and $450 \mathrm{~cm}^{-1}$. Journal of the Optical Society of America 1977, 67, 902.

(14) Draegert, D. A.; Stone, N. W. B.; Curnutte, B.; Williams, D. Far-Infrared Spectrum of Liquid Water. Journal of the Optical Society of America 1966, 56, 64.

(15) Sharma, M.; Resta, R.; Car, R. Intermolecular Dynamical Charge Fluctuations in Water: A Signature of the H-Bond Network. Physical Review Letters 2005, 95, 187401.

(16) Heyden, M.; Sun, J.; Funkner, S.; Mathias, G.; Forbert, H.; Havenith, M.; Marx, D. Dissecting the $\mathrm{THz}$ spectrum of liquid water from first principles via correlations in time and space. Proceedings of the National Academy of Sciences 2010, 10\%, 1206812073.

(17) Torii, H. Cooperative Contributions of the Intermolecular Charge Fluxes and Intramolecular Polarizations in the Far-Infrared Spectral Intensities of Liquid Water. Journal of Chemical Theory and Computation 2014, 10, 1219-1227.

(18) Torii, H. Intermolecular Electron Density Modulations in Water and Their Effects on the Far-Infrared Spectral Profiles at $6 \mathrm{THz}$. The Journal of Physical Chemistry B 2011, 115, 6636-6643.

(19) Torii, H. Dynamical behavior of molecular partial charges implied by the far-infrared spectral profile of liquid water. Chemical Physics 2018, 512, 165-170. 
(20) Ito, H.; Hasegawa, T.; Tanimura, Y. Effects of Intermolecular Charge Transfer in Liquid Water on Raman Spectra. The Journal of Physical Chemistry Letters 2016, 7, $4147-4151$.

(21) Castner, E. W.; Chang, Y. J.; Chu, Y. C.; Walrafen, G. E. The intermolecular dynamics of liquid water. The Journal of Chemical Physics 1995, 102, 653-659.

(22) Sidler, D.; Meuwly, M.; Hamm, P. An efficient water force field calibrated against intermolecular THz and Raman spectra. The Journal of Chemical Physics 2018, 148, 244504.

(23) Berendsen, H. J. C.; Grigera, J. R.; Straatsma, T. P. The missing term in effective pair potentials. The Journal of Physical Chemistry 1987, 91, 6269-6271.

(24) Jorgensen, W. L.; Chandrasekhar, J.; Madura, J. D.; Impey, R. W.; Klein, M. L. Comparison of simple potential functions for simulating liquid water. The Journal of Chemical Physics 1983, 79, 926-935.

(25) Jorgensen, W. L.; Madura, J. D. Temperature and size dependence for Monte Carlo simulations of TIP4P water. Molecular Physics 1985, 56, 1381-1392.

(26) Mahoney, M. W.; Jorgensen, W. L. A five-site model for liquid water and the reproduction of the density anomaly by rigid, nonpolarizable potential functions. The Journal of Chemical Physics 2000, 112, 8910-8922.

(27) Abascal, J. L. F.; Vega, C. A general purpose model for the condensed phases of water: TIP4P/2005. The Journal of Chemical Physics 2005, 123, 234505.

(28) Abascal, J. L. F.; Sanz, E.; García Fernández, R.; Vega, C. A potential model for the study of ices and amorphous water: TIP4P/Ice. The Journal of Chemical Physics 2005, 122, 234511. 
(29) Vega, C.; Abascal, J. L. F. Simulating water with rigid non-polarizable models: a general perspective. Physical Chemistry Chemical Physics 2011, 13, 19663.

(30) Neumann, M. Dielectric relaxation in water. Computer simulations with the TIP4P potential. The Journal of Chemical Physics 1986, 85, 1567-1580.

(31) Zasetsky, A. Y.; Gaiduk, V. I. Study of Temperature Effect on Far-Infrared Spectra of Liquid $\mathrm{H}_{2} \mathrm{O}$ and $\mathrm{D}_{2} \mathrm{O}$ by Analytical Theory and Molecular Dynamic Simulations. The Journal of Physical Chemistry A 2007, 111, 5599-5606.

(32) Shi, L.; Ni, Y.; Drews, S. E. P.; Skinner, J. L. Dielectric constant and low-frequency infrared spectra for liquid water and ice Ih within the E3B model. The Journal of Chemical Physics 2014, 141, 084508.

(33) Sega, M.; Schröder, C. Dielectric and Terahertz Spectroscopy of Polarizable and Nonpolarizable Water Models: A Comparative Study. The Journal of Physical Chemistry A 2015, 119, 1539-1547.

(34) Rick, S. W.; Stuart, S. J. Reviews in Computational Chemistry, Volume 18; John Wiley \& Sons, Inc.: Hoboken, New Jersey, USA, pp 89-146.

(35) Demerdash, O.; Wang, L.; HeadGordon, T. Advanced models for water simulations. WIREs Computational Molecular Science 2018, 8, e1355.

(36) Wick, C. D.; Kuo, I.-F. W.; Mundy, C. J.; Dang, L. X. The Effect of Polarizability for Understanding the Molecular Structure of Aqueous Interfaces. Journal of Chemical Theory and Computation 2007, 3, 2002-2010.

(37) Kuo, I.-F. W.; Mundy, C. J.; Eggimann, B. L.; McGrath, M. J.; Siepmann, J. I.; Chen, B.; Vieceli, J.; Tobias, D. J. Structure and Dynamics of the Aqueous LiquidVapor Interface: A Comprehensive Particle-Based Simulation Study . The Journal of Physical Chemistry B 2006, 110, 3738-3746. 
(38) Cramer, C. J. Essentials of Computational Chemistry: Theories and Models, 2nd ed.; John Wiley \& Sons, Ltd: West Sussex, England, 2004; p 618.

(39) Meister, J.; Schwarz, W. H. E. Principal Components of Ionicity. The Journal of Physical Chemistry 1994, 98, 8245-8252.

(40) Wang, B.; Truhlar, D. G. Partial atomic charges and screened charge models of the electrostatic potential. Journal of Chemical Theory and Computation 2012, 8, 19891998.

(41) Zhao, D. X.; Zhao, J.; Zhu, Z. W.; Zhang, C.; Yang, Z. Z. A model of atoms in molecules based on potential acting on one electron in a molecule: I. Partition and atomic charges obtained from ab initio calculations. International Journal of Quantum Chemistry 2018, 118, 1.

(42) Mulliken, R. S. Electronic Population Analysis on LCAO-MO Molecular Wave Functions. I. The Journal of Chemical Physics 1955, 23, 1833-1840.

(43) Reed, A. E.; Weinstock, R. B.; Weinhold, F. Natural population analysis. The Journal of Chemical Physics 1985, 83, 735-746.

(44) Reed, A. E.; Curtiss, L. A.; Weinhold, F. Intermolecular interactions from a natural bond orbital, donor-acceptor viewpoint. Chemical Reviews 1988, 88, 899-926.

(45) Cusachs, L.; Politzer, P. On the problem of defining the charge on an atom in a molecule. Chemical Physics Letters 1968, 1, 529-531.

(46) Doggett, G. Calculation of effective atomic-charges from molecular orbital wavefunctions. Journal of the Chemical Society A: Inorganic, Physical, Theoretical 1969, 229.

(47) Fonseca Guerra, C.; Handgraaf, J.-W.; Baerends, E. J.; Bickelhaupt, F. M. Voronoi deformation density (VDD) charges: Assessment of the Mulliken, Bader, Hirshfeld, 
Weinhold, and VDD methods for charge analysis. Journal of Computational Chemistry 2004, 25, 189-210.

(48) Wang, B.; Li, S. L.; Truhlar, D. G. Modeling the Partial Atomic Charges in Inorganometallic Molecules and Solids and Charge Redistribution in Lithium-Ion Cathodes. Journal of Chemical Theory and Computation 2014, 10, 5640-5650.

(49) Breneman, C. M.; Wiberg, K. B. Determining atom-centered monopoles from molecular electrostatic potentials. The need for high sampling density in formamide conformational analysis. Journal of Computational Chemistry 1990, 11, 361-373.

(50) Salomon-Ferrer, R.; Case, D. A.; Walker, R. C. An overview of the Amber biomolecular simulation package. Wiley Interdisciplinary Reviews: Computational Molecular Science 2013, 3, 198-210.

(51) Wang, J.; Wolf, R. M.; Caldwell, J. W.; Kollman, P. A.; Case, D. A. Development and testing of a general amber force field. Journal of Computational Chemistry 2004, 25, $1157-1174$.

(52) Schauperl, M.; Nerenberg, P. S.; Jang, H.; Wang, L.-P.; Bayly, C. I.; Mobley, D. L.; Gilson, M. K. Non-bonded force field model with advanced restrained electrostatic potential charges (RESP2). Communications Chemistry 2020, 3, 44.

(53) Duan, Y.; Wu, C.; Chowdhury, S.; Lee, M. C.; Xiong, G.; Zhang, W.; Yang, R.; Cieplak, P.; Luo, R.; Lee, T. et al. A point-charge force field for molecular mechanics simulations of proteins based on condensed-phase quantum mechanical calculations. Journal of Computational Chemistry 2003, 24, 1999-2012.

(54) Henchman, R. H.; Essex, J. W. Generation of OPLS-like charges from molecular electrostatic potential using restraints. Journal of Computational Chemistry 1999, 20, $483-498$. 
(55) Sigfridsson, E.; Ryde, U. Comparison of methods for deriving atomic charges from the electrostatic potential and moments. Journal of Computational Chemistry 1998, 19, 377-395.

(56) Dognon, J.-P.; Durand, S.; Granucci, G.; Lévy, B.; Millié, P.; Rabbe, C. Atomic charges for molecular dynamics calculations. Journal of Molecular Structure: THEOCHEM 2000, 507, 17-23.

(57) Francl, M. M.; Carey, C.; Chirlian, L. E.; Gange, D. M. Charges fit to electrostatic potentials. II. Can atomic charges be unambiguously fit to electrostatic potentials? Journal of Computational Chemistry 1996, 17, 367-383.

(58) Singh, U. C.; Kollman, P. A. An approach to computing electrostatic charges for molecules. Journal of Computational Chemistry 1984, 5, 129-145.

(59) Bayly, C. I.; Cieplak, P.; Cornell, W.; Kollman, P. A. A well-behaved electrostatic potential based method using charge restraints for deriving atomic charges: the RESP model. The Journal of Physical Chemistry 1993, 97, 10269-10280.

(60) Hu, H.; Lu, Z.; Yang, W. Fitting Molecular Electrostatic Potentials from Quantum Mechanical Calculations. Journal of Chemical Theory and Computation 2007, 3, 10041013.

(61) Hirshfeld, F. L. Bonded-atom fragments for describing molecular charge densities. Theoretica Chimica Acta 1977, 44, 129-138.

(62) Bultinck, P.; Van Alsenoy, C.; Ayers, P. W.; Carbó-Dorca, R. Critical analysis and extension of the Hirshfeld atoms in molecules. The Journal of Chemical Physics 2007, 126, 144111.

(63) Geldof, D.; Krishtal, A.; Blockhuys, F.; Van Alsenoy, C. An Extension of the Hirshfeld 
Method to Open Shell Systems Using Fractional Occupations. Journal of Chemical Theory and Computation 2011, 7, 1328-1335.

(64) Ghillemijn, D.; Bultinck, P.; Van Neck, D.; Ayers, P. W. A self-consistent Hirshfeld method for the atom in the molecule based on minimization of information loss. Journal of Computational Chemistry 2011, 32, 1561-1567.

(65) Verstraelen, T.; Ayers, P. W.; Van Speybroeck, V.; Waroquier, M. Hirshfeld-E Partitioning: AIM Charges with an Improved Trade-off between Robustness and Accurate Electrostatics. Journal of Chemical Theory and Computation 2013, 9, 2221-2225.

(66) Lu, T.; Chen, F. Atomic Dipole Moment Corrected Hirshfeld Population Method. Journal of Theoretical and Computational Chemistry 2012, 11, 163-183.

(67) Marenich, A. V.; Jerome, S. V.; Cramer, C. J.; Truhlar, D. G. Charge model 5: An extension of hirshfeld population analysis for the accurate description of molecular interactions in gaseous and condensed phases. Journal of Chemical Theory and Computation 2012, 8, 527-541.

(68) Bader, R. F. W. Atoms in molecules. Accounts of Chemical Research 1985, 18, 9-15.

(69) Åstrand, P.-O.; Ruud, K.; Mikkelsen, K. V.; Helgaker, T. Atomic Charges of the Water Molecule and the Water Dimer. The Journal of Physical Chemistry A 1998, 102, 7686-7691.

(70) Martin, F.; Zipse, H. Charge distribution in the water molecule -A comparison of methods. Journal of Computational Chemistry 2005, 26, 97-105.

(71) Wiberg, K. B.; Rablen, P. R. Comparison of atomic charges derived via different procedures. Journal of Computational Chemistry 1993, 14, 1504-1518.

(72) D. Thompson, J.; D. Xidos, J.; M. Sonbuchner, T.; J. Cramer, C.; G. Truhlar, D. 
More reliable partial atomic charges when using diffuse basis sets. PhysChemComm 2002, 5, 117.

(73) Besler, B. H.; Merz, K. M.; Kollman, P. A. Atomic charges derived from semiempirical methods. Journal of Computational Chemistry 1990, 11, 431-439.

(74) Frisch, M. J.; Trucks, G. W.; Schlegel, H. B.; Scuseria, G. E.; Robb, M. A.; Cheeseman, J. R.; Scalmani, G.; Barone, V.; Petersson, G. A.; Nakatsuji, H. et al. Gaussian16 Revision C.01. 2016; Gaussian Inc. Wallingford CT.

(75) Becke, A. D. Density-functional exchange-energy approximation with correct asymptotic behavior. Physical Review A 1988, 38, 3098-3100.

(76) Lee, C.; Yang, W.; Parr, R. G. Development of the Colle-Salvetti correlation-energy formula into a functional of the electron density. Physical Review B 1988, 37, 785-789.

(77) Becke, A. D. Densityfunctional thermochemistry. III. The role of exact exchange. The Journal of Chemical Physics 1993, 98, 5648-5652.

(78) Zhao, Y.; Truhlar, D. G. The M06 suite of density functionals for main group thermochemistry, thermochemical kinetics, noncovalent interactions, excited states, and transition elements: two new functionals and systematic testing of four M06-class functionals and 12 other function. Theoretical Chemistry Accounts 2008, 120, 215241.

(79) Zhao, Y.; Truhlar, D. G. Density Functional for Spectroscopy: No Long-Range SelfInteraction Error, Good Performance for Rydberg and Charge-Transfer States, and Better Performance on Average than B3LYP for Ground States. The Journal of Physical Chemistry A 2006, 110, 13126-13130.

(80) Zhao, Y.; Truhlar, D. G. Comparative DFT Study of van der Waals Complexes: Rare- 
Gas Dimers, Alkaline-Earth Dimers, Zinc Dimer, and Zinc-Rare-Gas Dimers. The Journal of Physical Chemistry A 2006, 110, 5121-5129.

(81) Chai, J.-D.; Head-Gordon, M. Long-range corrected hybrid density functionals with damped atomatom dispersion corrections. Physical Chemistry Chemical Physics 2008, 10,6615 .

(82) Vydrov, O. A.; Scuseria, G. E. Assessment of a long-range corrected hybrid functional. The Journal of Chemical Physics 2006, 125, 234109.

(83) Grimme, S. Semiempirical hybrid density functional with perturbative second-order correlation. The Journal of Chemical Physics 2006, 124, 034108.

(84) Lu, T.; Chen, F. Multiwfn: A multifunctional wavefunction analyzer. Journal of Computational Chemistry 2012, 33, 580-592.

(85) Shao, Y.; Gan, Z.; Epifanovsky, E.; Gilbert, A. T.; Wormit, M.; Kussmann, J.; Lange, A. W.; Behn, A.; Deng, J.; Feng, X. et al. Advances in molecular quantum chemistry contained in the Q-Chem 4 program package. Molecular Physics 2015, 113, $184-215$.

(86) Hoy, A.; Bunker, P. A precise solution of the rotation bending Schrödinger equation for a triatomic molecule with application to the water molecule. Journal of Molecular Spectroscopy 1979, 74, 1-8.

(87) Miliordos, E.; Xantheas, S. S. An accurate and efficient computational protocol for obtaining the complete basis set limits of the binding energies of water clusters at the MP2 and $\operatorname{CCSD}(\mathrm{T})$ levels of theory: Application to $(\mathrm{H} 2 \mathrm{O}) \mathrm{m}, \mathrm{m}=2-6,8,11,16$, and 17. Journal of Chemical Physics 2015, 142.

(88) Dyke, T. R.; Mack, K. M.; Muenter, J. S. The structure of water dimer from molecular 
beam electric resonance spectroscopy. The Journal of Chemical Physics 1977, 66, $498-510$.

(89) Berendsen, H.; van der Spoel, D.; van Drunen, R. GROMACS: A message-passing parallel molecular dynamics implementation. Computer Physics Communications 1995, $91,43-56$.

(90) Abraham, M. J.; Murtola, T.; Schulz, R.; Páll, S.; Smith, J. C.; Hess, B.; Lindahl, E. GROMACS: High performance molecular simulations through multi-level parallelism from laptops to supercomputers. SoftwareX 2015, 1-2, 19-25.

(91) Hamm, P. 2D-Raman-THz spectroscopy: A sensitive test of polarizable water models. The Journal of Chemical Physics 2014, 141, 184201.

(92) Nosé, S. A unified formulation of the constant temperature molecular dynamics methods. The Journal of Chemical Physics 1984, 81, 511-519.

(93) Hoover, W. G. Canonical dynamics: Equilibrium phase-space distributions. Physical Review A 1985, 31, 1695-1697.

(94) Parrinello, M.; Rahman, A. Polymorphic transitions in single crystals: A new molecular dynamics method. Journal of Applied Physics 1981, 52, 7182-7190.

(95) Darden, T.; York, D.; Pedersen, L. Particle mesh Ewald: An N log( N ) method for Ewald sums in large systems. The Journal of Chemical Physics 1993, 98, 10089-10092.

(96) Essmann, U.; Perera, L.; Berkowitz, M. L.; Darden, T.; Lee, H.; Pedersen, L. G. A smooth particle mesh Ewald method. The Journal of Chemical Physics 1995, 103, $8577-8593$.

(97) Hait, D.; Head-Gordon, M. How Accurate Is Density Functional Theory at Predicting Dipole Moments? An Assessment Using a New Database of 200 Benchmark Values. Journal of Chemical Theory and Computation 2018, 14, 1969-1981. 
(98) Choudhuri, I.; Truhlar, D. G. Calculating and Characterizing the Charge Distributions in Solids. Journal of Chemical Theory and Computation 2020, 16, 5884-5892.

(99) Hickey, A. L.; Rowley, C. N. Benchmarking Quantum Chemical Methods for the Calculation of Molecular Dipole Moments and Polarizabilities. The Journal of Physical Chemistry A 2014, 118, 3678-3687.

(100) Davidson, E. R.; Chakravorty, S. A test of the Hirshfeld definition of atomic charges and moments. Theoretica Chimica Acta 1992, 83, 319-330.

(101) Fredin, L.; Nelander, B.; Ribbegård, G. Infrared spectrum of the water dimer in solid nitrogen. I. Assignment and force constant calculations. The Journal of Chemical Physics 1977, 66, 4065-4072.

(102) Huang, Z. S.; Miller, R. E. Highresolution nearinfrared spectroscopy of water dimer. The Journal of Chemical Physics 1989, 91, 6613-6631.

(103) Bouteiller, Y.; Tremblay, B.; Perchard, J. The vibrational spectrum of the water dimer: Comparison between anharmonic ab initio calculations and neon matrix infrared data between 14,000 and 90cm1. Chemical Physics 2011, 386, 29-40.

(104) Ronca, E.; Belpassi, L.; Tarantelli, F. A Quantitative View of Charge Transfer in the Hydrogen Bond: The Water Dimer Case. ChemPhysChem 2014, 15, 2682-2687.

(105) Mo, Y.; Gao, J.; Peyerimhoff, S. D. Energy decomposition analysis of intermolecular interactions using a block-localized wave function approach. The Journal of Chemical Physics 2000, 112, 5530-5538.

(106) De Silva, P.; Korchowiec, J. Energy partitioning scheme based on self-consistent method for subsystems: Populational space approach. Journal of Computational Chemistry 2011, 32, 1054-1064. 
(107) Khaliullin, R. Z.; Bell, A. T.; Head-Gordon, M. Electron Donation in the Water-Water Hydrogen Bond. Chemistry - A European Journal 2009, 15, 851-855.

(108) Autschbach, J.; Srebro, M. Delocalization Error and Functional Tuning in KohnSham Calculations of Molecular Properties. Accounts of Chemical Research 2014, 47, 25922602.

(109) Nauta, K.; Miller, R. E. Formation of Cyclic Water Hexamer in Liquid Helium: The Smallest Piece of Ice. Science 2000, 287, 293-295.

(110) Xantheas, S. S. Ab initio studies of cyclic water clusters $\left(\mathrm{H}_{2} \mathrm{O}\right)_{n}, \mathrm{n}=1-6$. III. Comparison of density functional with MP2 results. The Journal of Chemical Physics 1995, 102, 4505-4517.

(111) Speedy, R. J.; Madura, J. D.; Jorgensen, W. L. Network topology in simulated water. The Journal of Physical Chemistry 1987, 91, 909-913.

(112) Belch, A. C.; Rice, S. A. The distribution of rings of hydrogenbonded molecules in a model of liquid water. The Journal of Chemical Physics 1987, 86, 5676-5682.

(113) Temelso, B.; Archer, K. A.; Shields, G. C. Benchmark Structures and Binding Energies of Small Water Clusters with Anharmonicity Corrections. The Journal of Physical Chemistry A 2011, 115, 12034-12046.

(114) Tainter, C. J.; Skinner, J. L. The water hexamer: Three-body interactions, structures, energetics, and OH-stretch spectroscopy at finite temperature. The Journal of Chemical Physics 2012, 13\%, 104304.

(115) Smith, J. D.; Cappa, C. D.; Wilson, K. R.; Cohen, R. C.; Geissler, P. L.; Saykally, R. J. Unified description of temperature-dependent hydrogen-bond rearrangements in liquid water. Proceedings of the National Academy of Sciences 2005, 102, 14171-14174. 
(116) Ponder, J. W.; Case, D. A. Force Fields for Protein Simulations. Advances in Protein Chemistry 2003, 66, 27-85.

(117) Maier, J. A.; Martinez, C.; Kasavajhala, K.; Wickstrom, L.; Hauser, K. E.; Simmerling, C. ff14SB: Improving the Accuracy of Protein Side Chain and Backbone Parameters from ff99SB. Journal of Chemical Theory and Computation 2015, 11, 3696-3713.

(118) Horn, H. W.; Swope, W. C.; Pitera, J. W.; Madura, J. D.; Dick, T. J.; Hura, G. L.; Head-Gordon, T. Development of an improved four-site water model for biomolecular simulations: TIP4P-Ew. The Journal of Chemical Physics 2004, 120, 9665-9678.

(119) Fecko, C. J.; Loparo, J. J.; Roberts, S. T.; Tokmakoff, A. Local hydrogen bonding dynamics and collective reorganization in water: Ultrafast infrared spectroscopy of $\mathrm{HOD} / \mathrm{D}_{2} \mathrm{O}$. The Journal of Chemical Physics 2005, 122, 054506.

(120) Lee, A. J.; Rick, S. W. The effects of charge transfer on the properties of liquid water. The Journal of Chemical Physics 2011, 134, 184507.

(121) Bakó, I.; Daru, J.; Pothoczki, S.; Pusztai, L.; Hermansson, K. Effects of H-bond asymmetry on the electronic properties of liquid water An AIMD analysis. Journal of Molecular Liquids 2019, 293, 111579.

(122) Rick, S. W.; Stuart, S. J.; Berne, B. J. Dynamical fluctuating charge force fields: Application to liquid water. The Journal of Chemical Physics 1994, 101, 6141-6156.

(123) Silvestrelli, P. L.; Parrinello, M. Water Molecule Dipole in the Gas and in the Liquid Phase. Physical Review Letters 1999, 82, 3308-3311.

(124) Sharma, M.; Resta, R.; Car, R. Dipolar Correlations and the Dielectric Permittivity of Water. Physical Review Letters 2007, 98, 247401. 
(125) Ahlström, P.; Wallqvist, A.; Engström, S.; Jönsson, B. A molecular dynamics study of polarizable water. Molecular Physics 1989, 68, 563-581.

(126) Bernardo, D. N.; Ding, Y.; Krogh-Jespersen, K.; Levy, R. M. An Anisotropic Polarizable Water Model: Incorporation of All-Atom Polarizabilities into Molecular Mechanics Force Fields. The Journal of Physical Chemistry 1994, 98, 4180-4187.

(127) Bursulaya, B. D.; Jeon, J.; Zichi, D. A.; Kim, H. J. Generalized molecular mechanics including quantum electronic structure variation of polar solvents. II. A molecular dynamics simulation study of water. The Journal of Chemical Physics 1998, 108, 3286-3295.

(128) Site, L. D.; Alavi, A.; Lynden-Bell, R. M. The electrostatic properties of water molecules in condensed phases: an ab initio study. Molecular Physics 1999, 96, 16831693.

(129) Tu, Y.; Laaksonen, A. The electronic properties of water molecules in water clusters and liquid water. Chemical Physics Letters 2000, 329, 283-288.

(130) Harder, E.; Eaves, J. D.; Tokmakoff, A.; Berne, B. J. Polarizable molecules in the vibrational spectroscopy of water. Proceedings of the National Academy of Sciences 2005, 102, 11611-11616.

(131) Morrone, J. A.; Car, R. Nuclear Quantum Effects in Water. Physical Review Letters 2008, 101, 017801.

(132) Schmidt, J.; VandeVondele, J.; Kuo, I.-F. W.; Sebastiani, D.; Siepmann, J. I.; Hutter, J.; Mundy, C. J. IsobaricIsothermal Molecular Dynamics Simulations Utilizing Density Functional Theory: An Assessment of the Structure and Density of Water at Near-Ambient Conditions. The Journal of Physical Chemistry B 2009, 113, 1195911964. 
(133) Wang, L.; Ceriotti, M.; Markland, T. E. Quantum fluctuations and isotope effects in ab initio descriptions of water. The Journal of Chemical Physics 2014, 141, 104502.

(134) Lin, I.-C.; Seitsonen, A. P.; Tavernelli, I.; Rothlisberger, U. Structure and Dynamics of Liquid Water from ab Initio Molecular DynamicsComparison of BLYP, PBE, and revPBE Density Functionals with and without van der Waals Corrections. Journal of Chemical Theory and Computation 2012, 8, 3902-3910.

(135) Lee, H.-S.; Tuckerman, M. E. Dynamical properties of liquid water from ab initio molecular dynamics performed in the complete basis set limit. The Journal of Chemical Physics 2007, 126, 164501. 


\section{Graphical TOC Entry}

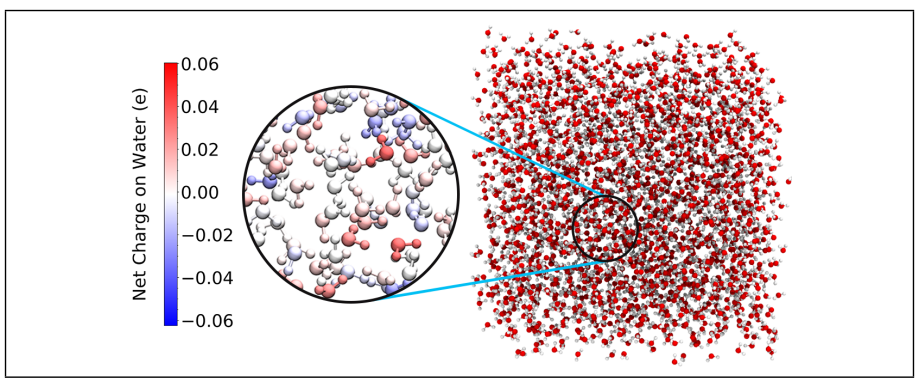

